\title{
PERFORMANCE COMPARISON OF BEAM FORMING TECHNIQUE USING LMS AND SMI ALGORITHMS
}

\author{
Rajiv A ${ }^{1 *}$ Lakshmi C $^{1}$ Divya $\mathrm{M}^{2}$ \\ ${ }^{1}$ Assistant Professor, ECE Sriram Engineering College, Perumalpattu -602 024 \\ ${ }^{2}$ Research Associate, Vellore Institute of Technology, Chennai \\ rajiva.ece@sriramec.edu.in,lakshmic.ece@sriramec.edu.in,divyamanne16@gmail.com
}

\begin{abstract}
The main objective is to analyse adaptive beam forming approach based on smart antenna. Several algorithms have been developed based on different criteria to compute the complex weights. In this paper the comparison of the performance of algorithms namely Least Mean Square algorithm (LMS) and Sample Matrix Inversion algorithm (SMI) are presented. The main advantage of these algorithms is its simplicity with a minimal loss of accuracy. This paper describes the design of an adaptive antenna array after receiving the signals from the desired and interfering directions. Then the weight vector is evaluated to minimize the error that provides an appropriate beam pattern to each subscriber. For these two algorithms the mean square error and Array factor are evaluated and compared. Simulation results revealed that Least Mean Squares (LMS) are best for beam forming (to form main lobes) towards desired user but they have limitations towards interference rejection. While Sample Matrix Inversion Algorithm (SMI) has satisfactory response towards beam forming and it gives better outcome for interference rejection. It is verified that convergence rate of SMI is faster than LMS so SMI is proved the best choice. The effect of changing number of elements on Array factor for SMI algorithm has also been studied.
\end{abstract}

Keywords - Antenna Arrays, Adaptive Algorithms, Beam forming, Interference, Smart antenna, Least Mean Squares (LMS), Sample Matrix Inversion Algorithm (SMI).

\section{INTRODUCTION}

Adaptive Beamforming is a technique in which an array of antennas is exploited to achieve maximum reception in a specified direction by estimating the signal arrival from a desired direction (in the presence of noise) while signals of the same frequency from other directions are rejected. This is achieved by varying the weights of each of the sensors (antennas) used in the array. It basically uses the idea that, though the signals emanating from different transmitters occupy the same frequency channel, they still arrive from different directions. The spatial separation is exploited to separate the desired signal from the interfering signals. A smart antenna system combines multiple antenna elements with a signal- processing capability to optimize its radiation and reception pattern automatically in response to the signal environment[3]. Least Mean Square (LMS) adaptive beam forming algorithm was developed for smart antenna application. There are a number of criteria for choosing the algorithm that will produce the optimum weights, they are closely relate to each other and therefore the choice of the one is critical in terms of its performance. The most mainly used adaptive algorithms include LMS (Least Mean Square) and SMI (sample Matrix Inverse) [5].
They have their own disadvantages and advantages as far as complexity, convergence, speed and other aspects are concerned.

\section{SMART ANTENNA}

Smart antenna (also known as adaptive array antenna) refers to a system of antenna arrays with smart signal processing algorithms that are used to identify spatial signature such as direction of arrival (DOA) of the signal and it is used to calculate beamforming vectors, to track and locate the antenna beam on the mobile/target.

The two important categories of smart antennas are:

\section{A. Switched Beam Antennas}

A switched beam antenna system forms multiple fixed beams with heightened sensitivity in particular directions. These antenna systems detect signal strength, choose from one of several predetermined, fixed beams, and switch from one beam to another as the mobile moves throughout the sector. 


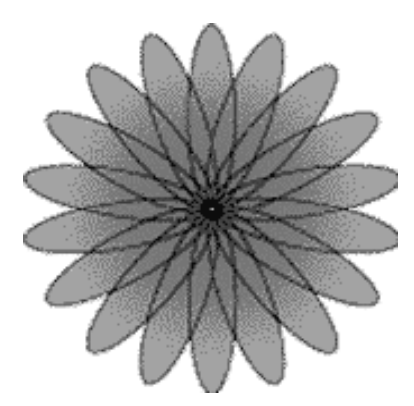

Figure.1 Switched beam

\section{B. Adaptive Array Antenna}

Only a small portion of the power transmitted in an omni directional manner is actually received by the intended user, while at the same time the rest, the 'wasted' power causes harmful interference to other potential users as shown in figure (2).Hence it is obvious that the omni directional power transmission is inefficient both in terms of power and capacity. Exploiting the adaptive antenna spatial filtering properties, it is possible to confine the radio energy associated with a given user to a small addressed volume, thus reducing the interference from others.

Adaptive array antenna technology represents the most advanced smart antenna approach. Using a variety of new signal-processing algorithms, the adaptive system takes advantage of its ability to effectively locate and track various types of signals to dynamically minimize interference and maximize intended signal reception[8].

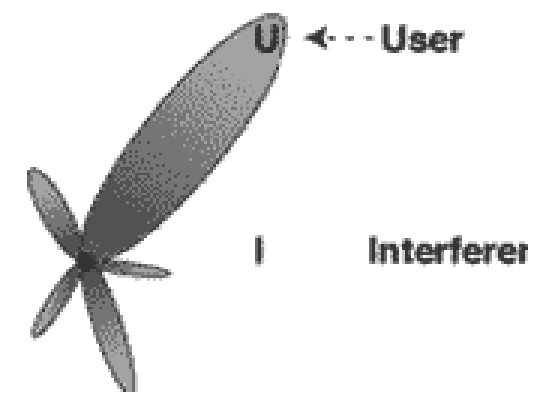

Figure.2 Adaptive Array

\section{A D A P T I VE BEAM FORMING}

The purpose of beamforming is to form a multiple beams towards desired users while nulling the interferers at the same time, through the adjustment of the beam former's weight vectors. It is the process of altering the complex weight onthe-fly to maximize the quality of the communication channel. Figure (3) shows a generic adaptive beamforming system which requires a reference signal.

The signal $\mathrm{x}(\mathrm{n})$ received by multiple antenna elements is multiplied with the coefficients in a weight vector ' $w$ ' which adjust the phase and amplitude of the incoming signal accordingly. This weighted signal is summed up, resulting in the array output $y(n)$. An adaptive algorithm is then employed to minimize the error $e(n)$ between a desired signal $d(n)$ and the array output $y(n)$.The complex weights wk for the antenna elements are carefully chosen to give the desired peaks and nulls in the radiation pattern of the array. The weights could then be slowly varied to steer the beam until maximum signal strength occurs and the direction to the signal source is found[3].

The use of multi-element antenna systems on both sides of the communication link has been proposed for extra ordinary increase of transmission rates. Adaptive algorithms require less priori knowledge of the signal characteristics such as interference directions of arrival, bandwidth and also require high computational effort.

Linearly constrained LMS algorithm is the most widely used algorithm due to its simplicity, generally efficient and robust. It requires only the knowledge and the bandwidth of the signal.

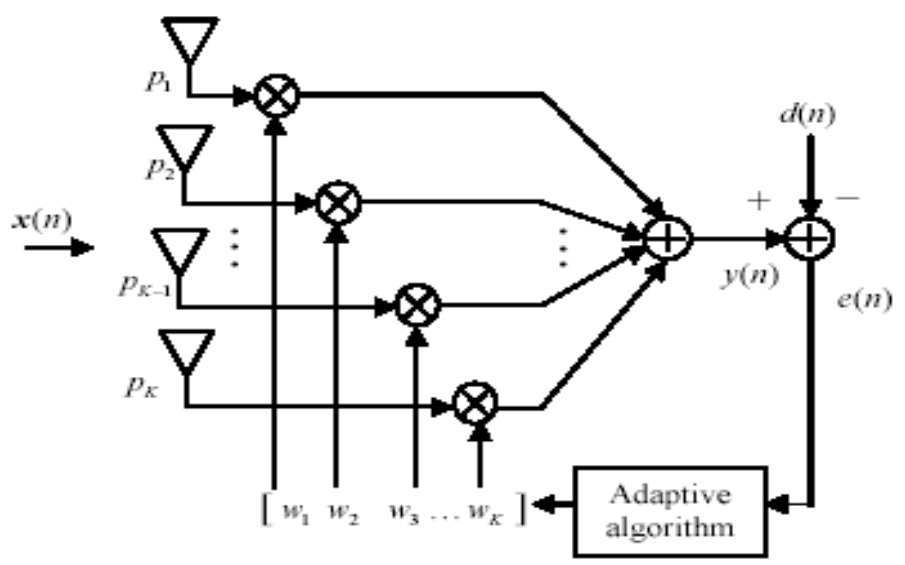

Figure.3 Block diagram of Adaptive beam forming 


\section{LEAST MEAN SQUARE}

\section{A. Introduction}

The Least Mean Square (LMS) algorithm, introduced by Widrow and Hoff in 1959, is an adaptive algorithm, which uses a gradient-based method of steepest decent. LMS algorithm uses the estimates of the gradient vector from the available data. LMS incorporates an iterative procedure that makes successive corrections to the weight vector in the direction of the negative of the gradient vector which eventually leads to the minimum mean square error. Compared to other algorithms LMS algorithm is relatively simple, it does not require correlation function calculation nor does it require matrix inversions[1].

\section{B. LSM Algorithms}

Consider a Uniform Linear Array (ULA) with $N$ isotropic elements, which forms the integral part of the adaptive beamforming system as shown in the figure 4 . The output of the antenna array $\mathrm{x}(\mathrm{t})$ is given by equation (1) [3],

$$
\begin{aligned}
& \mathrm{N}_{\mathrm{u}}
\end{aligned}
$$

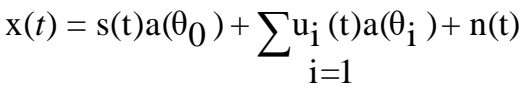

The weights here will be computed using LMS algorithms based on Minimum Squared Error (MSE) criterion.

Therefore the weight update can be given by the following equations,

$$
\begin{aligned}
\mathrm{w}(\mathrm{n}+1)= & \mathrm{w}(\mathrm{n})+\mu \mathrm{x}(\mathrm{n})\left[\mathrm{d}^{*}(\mathrm{n})-\mathrm{x}^{\wedge} \mathrm{h}(\mathrm{n}) \mathrm{w}(\mathrm{n})\right] \\
& =\mathrm{w}(\mathrm{n})+\mu \mathrm{x}(\mathrm{n}) \mathrm{e}^{*}(\mathrm{n})
\end{aligned}
$$

Where $\mu$ is the step-size parameter and controls the convergence characteristics of the LMS algorithm.

The LMS algorithm is initiated with an arbitrary value w (0) for the weight vector at $n=0$. The successive corrections of the weight vector eventually leads to the minimum value of the mean squared error.

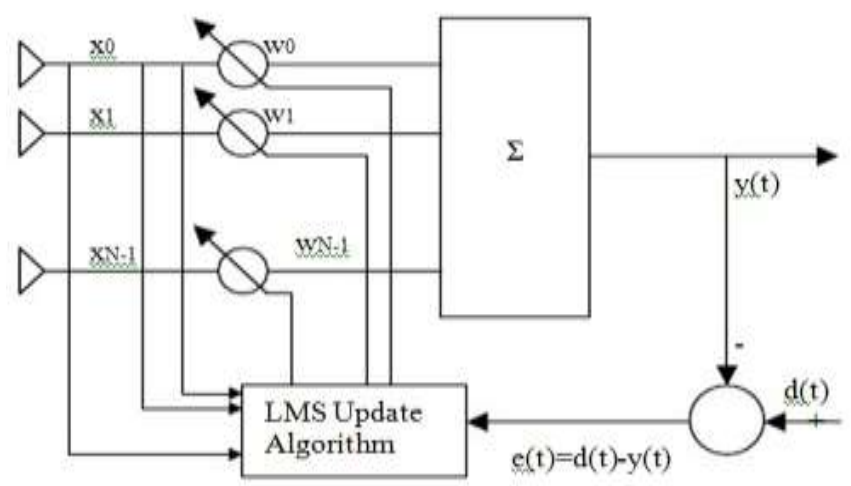

Figure. 4 LMS Adaptive Beamforming Network

\section{Simulation Results for LMS}

For simulation purpose a 5-element linear array is used with its individual elements spaced at half wave length distance $(\lambda / 2)$.

The desired signal s (t) arriving at $\theta_{0}$ is a simple complex sinusoidal phase modulated signal of the form given in equation (4),

$$
s(t)=e^{j \sin (\omega t)}
$$

The interfering signals $u_{i}(t)$ arriving at angles $\theta_{i}$ is also in the above form.

By doing so it can be shown in the simulations how interfering signals of the same frequency as the desired signal can be separated to achieve rejection of co-channel interference.

For simplicity purpose the reference signal $d(t)$ is considered to be the same as the desired signal $s(t)$. The array factor is obtained for the AOA of the desired user is at 30 degree and an interferer at 60 degree. The figure (5) shows how the LMS algorithm is able to update the weights block-wise to force deep nulls in the direction of the interferer and maximum in the direction of the desired signal. The optimum weight vector that the algorithm converged is found to be $w 1=0.3279+$ $0.0051 \mathrm{i}, \mathrm{w} 2=0.1599-0.1654 \mathrm{i} ; \mathrm{w} 3=-0.1678-0.1652 \mathrm{i}$ and $\mathrm{w} 4=0.0063+0.3125 \mathrm{i}$. 


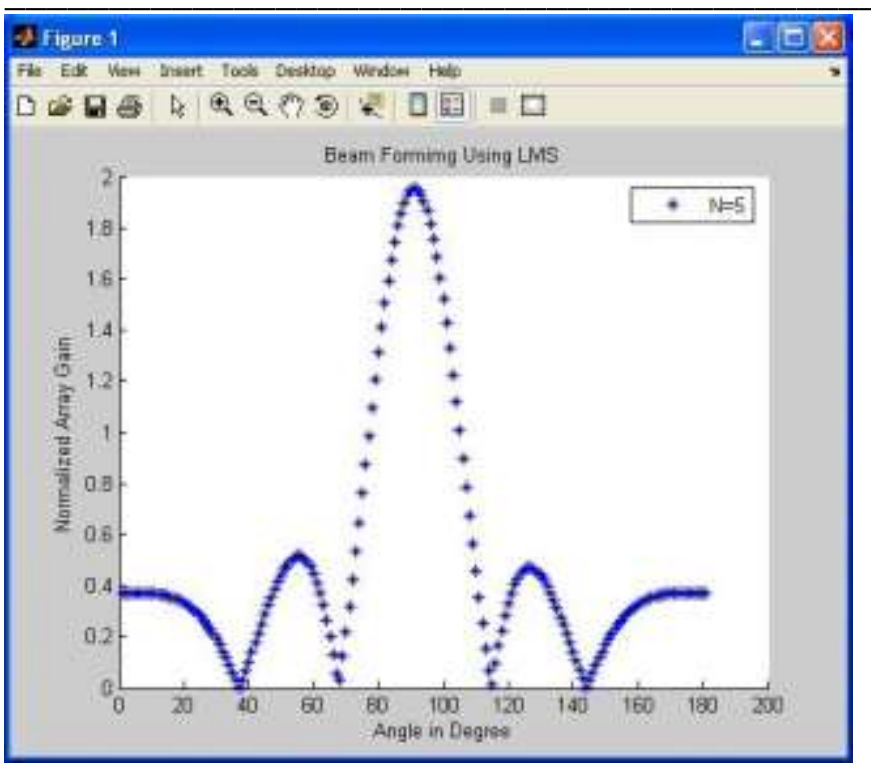

Figure. 5 Normalized array factor plot for LMS Algorithm with AOA for desired user is 30 degree and 60 degree for interfere with constant space of $\lambda / 2$ between elements.

The LMS error plot in figure (6) shows that the LMS algorithm converges. In this case the LMS error is almost 0.12 at around 5000 samples.

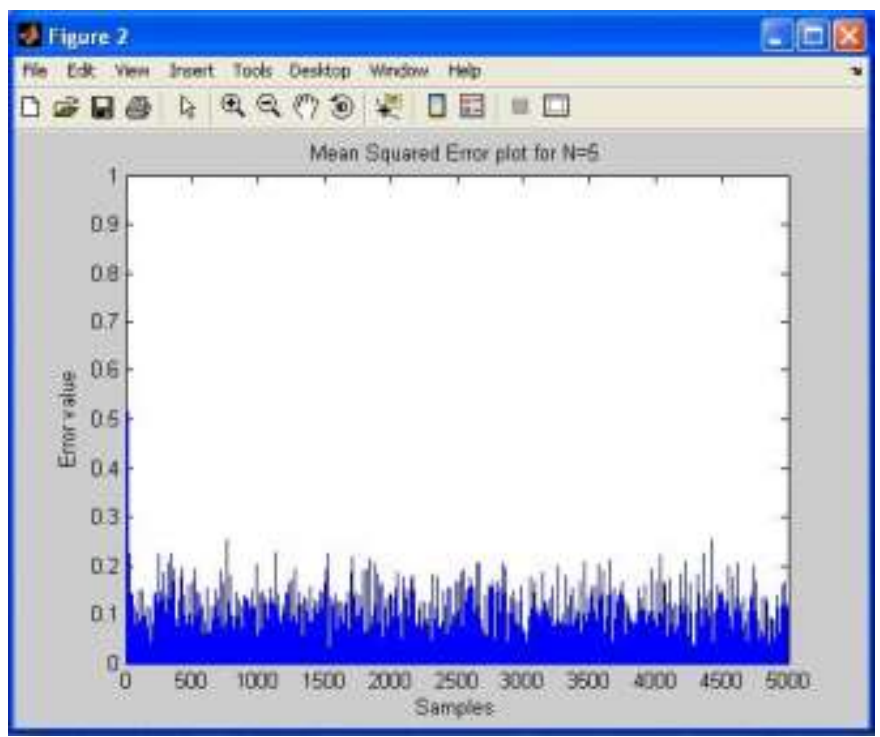

Figure. 6 Mean square error plot for LMS Algorithms

\section{SAMPLE MATRIX INVERSION}

\section{A. Introduction}

The LMS algorithm is a continuously adaptive algorithm and has a slow convergence when the eigen values of the covariance matrix are widespread. When the transmission is discontinuous, a block adaptive approach would give a better performance than a continuous approach. One such algorithm is the Sample Matrix Inversion (SMI), which provides good performance in a discontinuous traffic. However, it requires that the number of interferers and their positions remain constant during the duration of the block acquisition.

\section{B. SMI Algorithms}

The equations for the covariance matrix $R$ and the correlation matrix r, is given as,

$$
\begin{aligned}
& \mathrm{R}=\mathrm{E}\left[\mathrm{x}(\mathrm{t}) \mathrm{x}^{\mathrm{H}}(\mathrm{t})\right] \\
& \mathrm{r}=\mathrm{E}[\mathrm{d}(\mathrm{t}) \mathrm{x}(\mathrm{t})]
\end{aligned}
$$

If a priori information about the desired and the interfering signals is known, then the optimum weights can be calculated directly by using the Weiner solution,

$$
\mathrm{w}_{\mathrm{opt}}=\mathrm{r} / \mathrm{R}
$$

However, in practice signals are not known and the signal environment keeps changing. Therefore optimal weights can be computed by obtaining the estimates of the covariance matrix $\mathrm{R}$ and the correlation matrix $\mathrm{r}$, by time averaging from the block of input data [11].

The estimates of the matrices over a block size N2 - N1 are given by equations (8) and (9),

$$
\begin{gathered}
\hat{R}=\sum_{\mathrm{i}=N_{1}}^{N_{2}} \mathrm{x}^{\mathrm{i}) \mathrm{x}^{\mathrm{H}}(\mathrm{i})} \\
\hat{r}=\sum_{\mathrm{i}=N_{1}}^{N_{2}}{ }^{*}(\mathrm{i}) \mathrm{x}^{\mathrm{H}}(\mathrm{i})
\end{gathered}
$$

Where $N_{1}$ and $N_{2}$ forms the lower and the upper limit of the observation interval. The weight vector can now be estimated 
by the equation (10),

$$
\hat{w}=\hat{R}^{-1} \hat{r}
$$

There is always a residual error in the SMI algorithm since it is based on estimation. The error e due to estimates can be calculated by the equation (11),

$$
\mathrm{e}=\hat{R} \mathrm{w}_{\mathrm{opt}}-\hat{r}
$$

The stability of the SMI algorithm depends on the ability to invert the large covariance matrix. In order to avoid a singularity of the covariance matrix, a zero- mean white Gaussian noise is added to the array response vector. It creates a strong additive component to the diagonal of the matrix. In the absence of noise in the system, a singularity occurs when the number of signals to be resolved is less than the number of elements in the array.

Since SMI employs direct matrix inversion the convergence of this algorithm is much faster compared to the LMS algorithm [12].

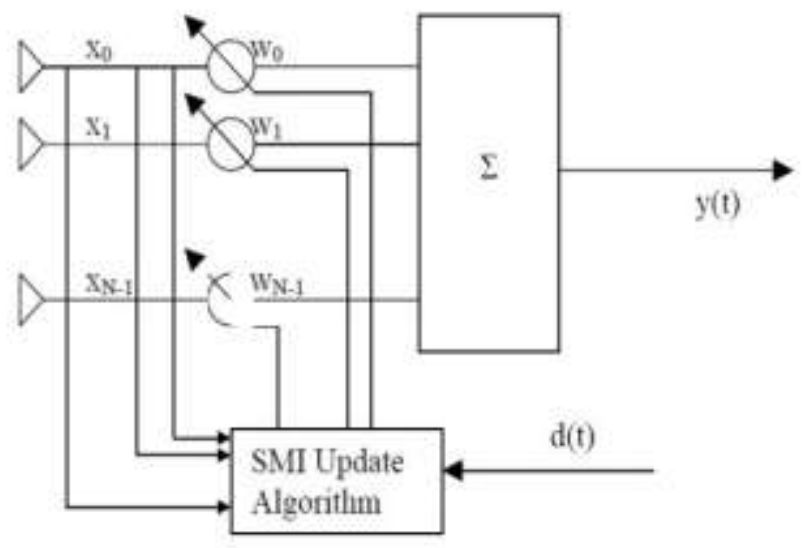

Figure. 7 SMI Adaptive Beamforming Network

\section{Simulation Results for SMI}

For simulation purposes, a similar scenario is considered as with the LMS simulation discussed in the above case. The SMI algorithm discussed here uses the Block Adaptation approach, the size of the block being equal to 10 time samples.

A 5-element linear array is used with its individual elements spaced at half-wavelength distance. The desired signal $s(t)$ arriving at $\theta_{0}$ is a simple sinusoidal-phase modulated signal of the same form as in equation (12),

$$
s(t)=e^{j \sin (\omega t)}
$$

The interfering signals $u_{i}(t)$ arriving at angles $\theta_{i}$ is also of the same form. By doing so it can be shown in the simulations how interfering signals of the same frequency as the desired signal can be separated to achieve rejection of co-channel interference.

The desired angle is arriving at 30 degrees and interfering signals arriving at angle of 60 degree. The array factor plot in figure (8) shows that the SMI algorithm is able to update the weights block wise to force deep nulls in the direction of the interferers and achieve a maximum in the direction of the desired signal. The optimum weight vectors for which the algorithm converged were found to be $\mathrm{w} 1=0.3093-0.1259 \mathrm{i}$, $\mathrm{w} 2=-0.2315-0.0760 \mathrm{i} ; \mathrm{w} 3=-0.2299-0.0996 \mathrm{i}$ and $\mathrm{w} 4$ $=0.1478+0.2925 \mathrm{i}$.

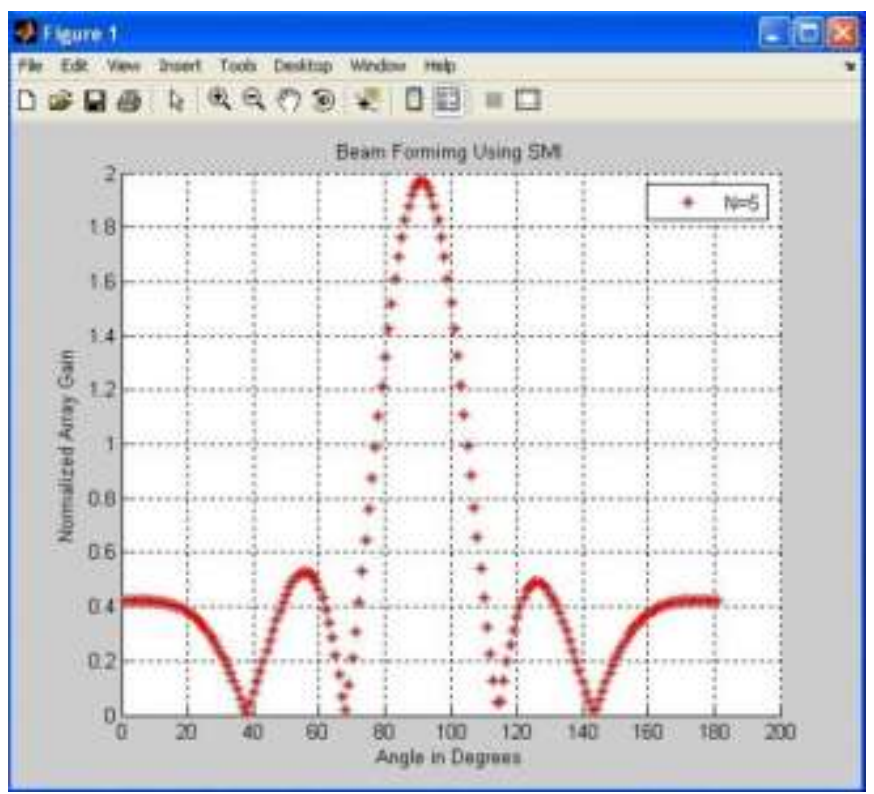

Figure. 8 Normalized array factor plot for SMI Algorithm with AOA for desired user is 30 degree and 60 degree for interfere with constant space of $\lambda / 2$ between elements.

The error plot for this situation is shown in figure (9). When compared to the LMS error SMI error is small. However, this error is uniform and is present throughout the acquisitions. This is because SMI does not require the error information to update the weights. These observations indicate that SMI algorithm converges almost immediately during the first block itself but there is a small amount of residual error throughout. 


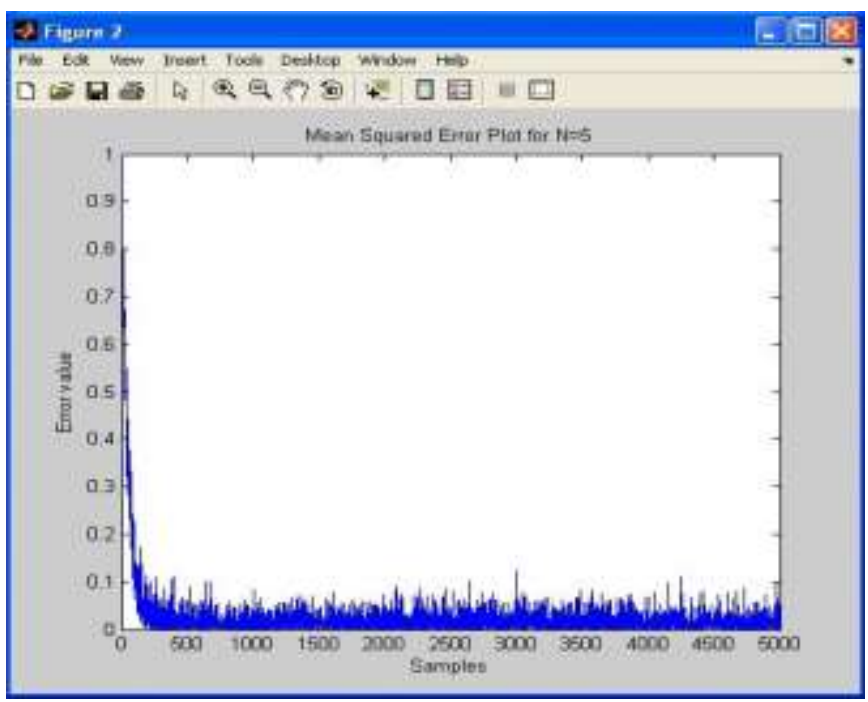

Figure. 9 Mean square error plot for SMI Algorithms

D. Effect of Number of Elements on Array Factor for SMI

Uniform linear array is taken for simulation purpose with spacing between array elements as $\lambda / 2$. AOA for desired user is set at 30 degree and interfering signals arriving at angle of 60 degree. The figure (10) shows how the SMI algorithm is able to update the weights block-wise to force deep nulls in the direction of the interferer and maximum in the direction of the desired signal. It also shows the normalized array factor for the number of elements $\mathrm{N}=5,8$ and 10 with spacing between the elements $d=0.5$ lambda.

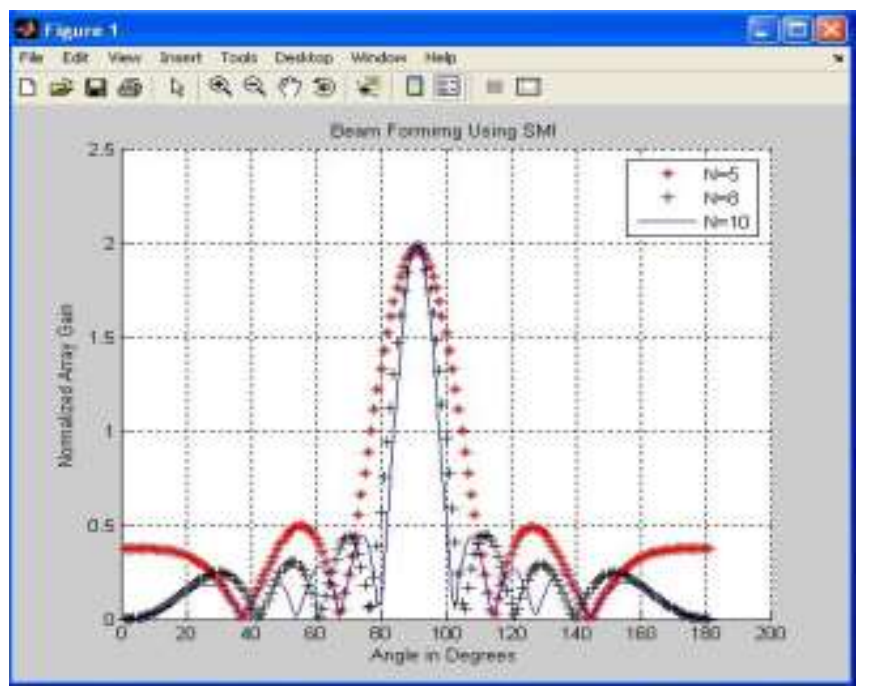

Figure.10 Normalized array factor plot for SMI Algorithm with AOA for desired user is 30 degree and 60 degree for interfere with constant space of $\lambda / 2$ between elements.

\section{E. Effect of Number of Elements Spacing on Array Factor for $S M I$}

A 5-element linear array is used with its individual elements spaced at $\mathrm{d}=0.5$ lambda, $\mathrm{d}=0.25, \mathrm{~d}=0.125$ lambda. For simulation purpose AOA for desired user is set at 30 degree and interfering signals arriving at angle of 60 degree. The figure (11) shows, how the SMI algorithm is able to update the weights block-wise to force deep nulls in the direction of the interferer and maximum in the direction of the desired signal. It also shows the normalized array factor for the elements $\mathrm{N}=5$, with spacing between the elements $d=0.5$ lambda, $\mathrm{d}=0.25, \mathrm{~d}=0.125$ lambda.

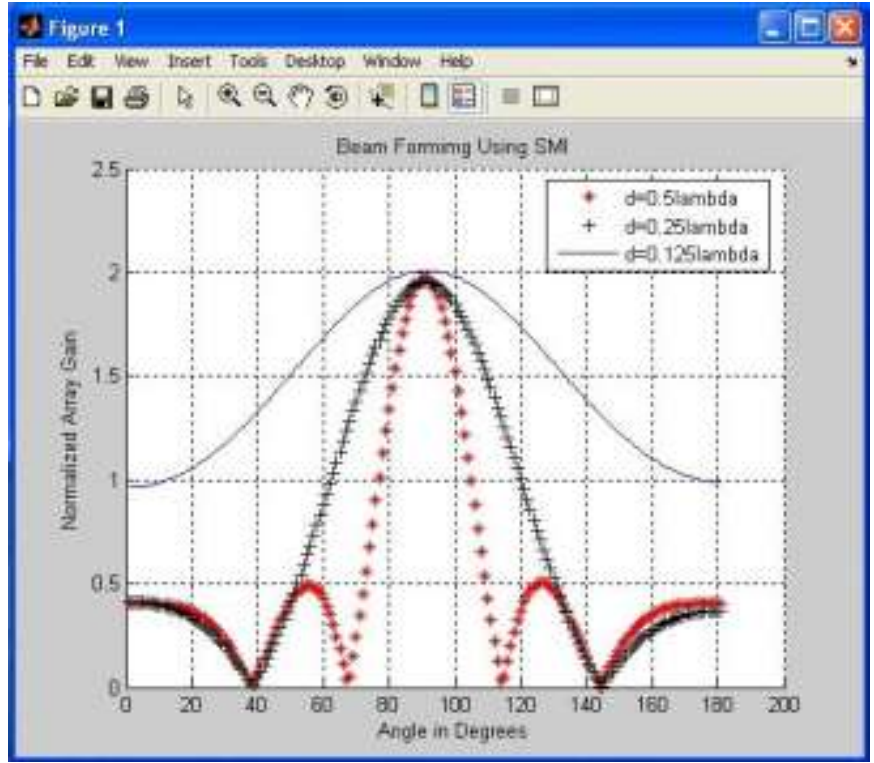

Figure 11 Normalized array factor plot for SMI Algorithm with AOA for desired user is 30 degree and 60 degree for interfere with different space between elements.

\section{RESULTS AND DISCUSSION}

The number of elements considered for simulation environment is 5 .

The LMS algorithm continuously adaptive algorithm and has a slow convergence when the Eigen values of the covariance matrix are widespread and hence the convergence takes much more time (figure 6). 
In block adaptive approach, that is, SMI algorithm would give better performance than LMS algorithms (figure 9), has several drawbacks. The correlation matrix may be ill conditioned resulting in error or singularities when inverted. In addition for large arrays, there is the challenge of inverting large matrix. To invert, the correlation matrix requires complex multiplication.

The SMI approach is used to speed up convergence rate, but both algorithms needs reference signals. SMI algorithm is computational more complex than the LMS algorithm.

Additional results are provided above to demonstrate the effect of number of array elements on array factor of SMI for approaches to better performance than the LMS algorithm. For simulation purpose uniform linear array of number of elements $\mathrm{N}=5,8$ and 10 with spacing between array elements as $\lambda / 2$, AOA for desired user is set at 30 degree and interfering signal arriving at angle of 60 degree is shown in figure (10). Also the other graph shown in figure (11) represents the effect of element spacing on the normalized array factor.

From above results we have proved that the SMI algorithm is better than LMS algorithms, with very narrower HPBW, for smart base station antenna for cellular communication networks.

\section{CONCLUSION}

This paper discussed various adaptive beamforming algorithms like Least Mean Square algorithms (LMS) and Sample matrix inversion algorithm (SMI) which are used in smart antennas. The result obtained from the simulations showed that the LMS had poor convergence compared to SMI, and the SMI algorithm is the most efficient algorithm.
WCDMA Communication Systems," IEEE Transactions on Vehicular Technology, Volume 52, Issue 4, July 2003, Pages 752760.

[7] J.H. Winters, "Smart Antenna Techniques and Their Application to Wireless Ad-hoc Networks," IEEE Transactions on Wireless Communications, Volume13, Issue 4, August 2006, Pages 77-83.

[8] S. Das, "Smart Antenna Design for wireless Communication using Adaptive Beam-forming Approach" IEEE Int. Conf. TENCON, 2008.

[9] Ch.Santhi Rani, "Smart Antenna Algorithms for WCDMA Mobile Communication Systems," IJCSNS, Vol.8, No.7, July 2008, Pages $182-186$.

[10] K.Meena alias Jeyanthi, "A Simple Adaptive Beam Forming Algorithm with interference suppression," IJET, Vol.1, No.1, April 2009, Pages 67-70.

[11] K.R.Shankar Kumar, "Performance Analysis of Adaptive Beam Forming for Microstrip Smart Antennas," IJCSCT, Vol.2, No.1, July 2009, Pages 305-309.

[12] M. Yasin, "Performance Analysis of LMS and NLMS Algorithms for a smart Antenna System", International J. Computer Application (0975-8887), 4(9): 25-32, 2010.

[13] Ch.Raghavendra, "Adaptive Beam Forming using DMI and CMA Algorithms," IJAEST, Vol.6 Issue No.1, July 2011, Pages 35-40.

[14] M. Yasin, "Enhanced Sample Matrix Inversion Is a Better for Smart Antenna System," World Applied Science Journal 10(10): 1167-1175, 2010, ISSN 1818-4952.

[15] Muhammad Salman Razzaq, "Performance Comparison of Adaptive beamforming Algorithms for Smart Antenna System", World Applied Science Journal 11 (07): 775-785, 2010, ISSN 1818-4952.

\section{REFERENCES}

[1] Simon Haykin, "Adaptive Filter Theory", Fourth edition, Pearson Education, Asia, 2002.

[2] Theodore, S. Rappaport, "Wireless Communications: Principles \& Practice", Second Edition, Pearson Education/ Prentice Hall of India, Third Indian Reprint 2003.

[3] Constantine A.Balanis, 'Antenna Theory: Analysis and Design', Second edition, John Wiley \& Sons Inc., 2005.

[4] Jeffrey H.Reed, 'Software Defined radio', Pearson education.

[5] L.C. Godara, "Application of Antenna Arrays to Mobile Communications. II. Beamforming and Direction-of-Arrival Considerations," Proceedings of IEEE, Volume 85, Issue 8 , August 1997, Pages 1195- 1245.

[6] H. Li and T. Liu, "Comparison of Beamforming Techniques for 